\title{
Principal component analysis of d-prime values from sensory discrimination tests using binary paired comparisons
}

\author{
Linander, Christine Borgen; Christensen, Rune Haubo Bojesen; Cleaver, Graham; Brockhoff, Per Bruun
}

Published in:

Food Quality and Preference

Link to article, DOI:

10.1016/j.foodqual.2019.103864

Publication date:

2020

Document Version

Early version, also known as pre-print

Link back to DTU Orbit

Citation (APA):

Linander, C. B., Christensen, R. H. B., Cleaver, G., \& Brockhoff, P. B. (2020). Principal component analysis of dprime values from sensory discrimination tests using binary paired comparisons. Food Quality and Preference, 81, [103864]. https://doi.org/10.1016/j.foodqual.2019.103864

\section{General rights}

Copyright and moral rights for the publications made accessible in the public portal are retained by the authors and/or other copyright owners and it is a condition of accessing publications that users recognise and abide by the legal requirements associated with these rights.

- Users may download and print one copy of any publication from the public portal for the purpose of private study or research.

- You may not further distribute the material or use it for any profit-making activity or commercial gain

- You may freely distribute the URL identifying the publication in the public portal 


\section{Journal Pre-proofs}

Principal component analysis of d-prime values from sensory discrimination tests using binary paired comparisons

Christine Borgen Linander, Rune Haubo Bojesen Christensen, Graham Cleaver,

Per Bruun Brockhoff

PII:

S0950-3293(18)30812-7

DOI: https://doi.org/10.1016/j.foodqual.2019.103864

Reference:

FQAP 103864

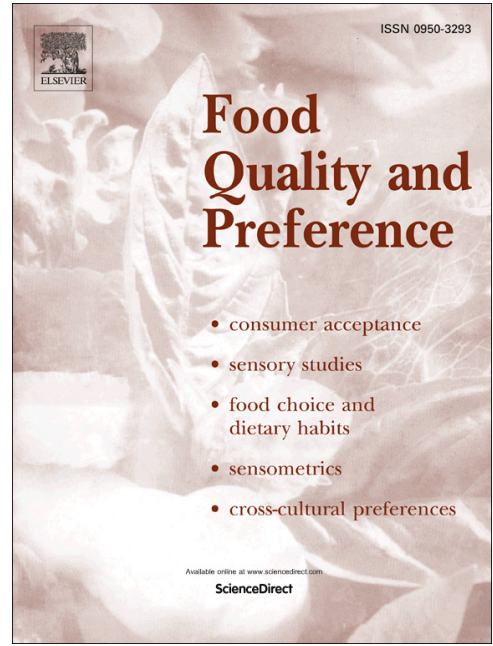

To appear in:

Food Quality and Preference

Received Date: $\quad 28$ September 2018

Accepted Date: $\quad 9$ December 2019

Please cite this article as: Linander, C.B., Bojesen Christensen, R.H., Cleaver, G., Brockhoff, P.B., Principal component analysis of d-prime values from sensory discrimination tests using binary paired comparisons, Food Quality and Preference (2019), doi: https://doi.org/10.1016/j.foodqual.2019.103864

This is a PDF file of an article that has undergone enhancements after acceptance, such as the addition of a cover page and metadata, and formatting for readability, but it is not yet the definitive version of record. This version will undergo additional copyediting, typesetting and review before it is published in its final form, but we are providing this version to give early visibility of the article. Please note that, during the production process, errors may be discovered which could affect the content, and all legal disclaimers that apply to the journal pertain.

(C) 2019 Published by Elsevier Ltd. 
8

\title{
Principal component analysis of d-prime values from sensory discrimination tests using binary paired comparisons
}

\author{
Christine Borgen Linander ${ }^{\mathrm{a}, \mathrm{b}, *}$, Rune Haubo Bojesen Christensen ${ }^{\mathrm{a}, \mathrm{c}}$, Graham Cleaver $^{\mathrm{d}}$, Per Bruun Brockhoff ${ }^{\mathrm{a}}$ \\ ${ }^{a}$ DTU Compute, Section of Statistics and Data Analysis, Technical University of Denmark, Richard Petersens Plads, Building 324, DK-2800 \\ Kongens Lyngby, Denmark \\ ${ }^{b}$ Clinical Research Center, Copenhagen University Hospital, Hvidovre, Kettegård Alle 30, DK-2650 Hvidovre, Denmark \\ ${ }^{c}$ Christensen Statistics, Bringetoften 7, DK-3500 Varlose, Denmark \\ ${ }^{d}$ Unilever Research and Development, Port Sunlight, Wirral, UK, CH63 $3 \mathrm{JW}$ (retired)
}

\begin{abstract}
When considering sensory discrimination studies, multiple d-prime values are often obtained from several sensory attributes. In this paper, we introduce principal component analysis as a way of gaining information about d-prime values across sensory attributes. Specifically, we propose estimating d-prime values using a Thurstonian mixed model for binary paired comparison data and then using these estimates in a principal component analysis. Binary paired comparisons are a sensitive way to test products with only subtle differences. When analyzing data with a Thurstonian mixed model, product-specific as well as assessor-specific d-prime values are obtained. Principal component analysis of these values results in information about products and assessors across multiple sensory attributes illustrated by product and attribute maps. Furthermore, the analysis captures individual differences. Thus, by using d-prime values from a multi-attribute 2-AFC study in principal component analysis insights that are typically obtained considering quantitative descriptive analysis are obtained.
\end{abstract}

Keywords: d-prime values, discrimination testing, assessor information, multi-product setting, Principal Component Analysis

\section{Introduction}

One could ask the question: is it meaningful to make a multi-attribute 2-AFC study when it is possible to consider standard profiling e.g. quantitative descriptive analysis (QDA)? In short, our answer is yes. Discrimination testing, including the 2-AFC test, is sometimes preferred because it brings value in its own right or simply because specific comparisons are of interest. Furthermore, by conducting a multi-attribute 2-AFC study, it is possible to obtain the insights that are typically obtained in a QDA: product attribute maps (biplots) and individual differences. To achieve this it is necessary to use principal component analysis (PCA). PCA is a well-established type of analysis for QDA and the solid arguments for using PCA on QDA data are equivalently relevant for a multi-attribute 2-AFC study. QDA is a commonly used method in sensory science and Lawless \& Heymann (1998) gives a nice introduction to this topic. If there are many evaluations, fatigue could be an issue for the 2-AFC study. However, when designing a multi-attribute 2-AFC study, this aspect can be considered by e.g. having the assessors evaluate the samples over multiple sessions or even days.

In discrimination studies, several attributes can be considered. One approach is to do an analysis for one attribute at a time (e.g. Linander et al. (2019)). For other types of sensory data, e.g. sensory profiling data, many attributes can be present. Such data are often analyzed by Principal Component Analysis (PCA) (Næs \& Risvik (1996), Næs et al. (2010), Lawless \& Heymann (1998)). PCA is a well-known multivariate analysis that is used in many applications e.g. in Chemometrics (Varmuza \& Filzmoser (2009)) as well as sensory and consumer science.

When considering discrimination studies, an advantage of using $\delta$, the measure for sensory differences, is that $\delta$ does

${ }^{*}$ Corresponding author. E-mail address: christine.borgen.linander@ regionh.dk (C. B. Linander). 
not depend on the discrimination test protocol, see e.g. Ennis (1993). Thus, it is common to obtain d-prime values, the estimates of $\delta$, when analyzing discrimination studies. In the analysis of sensory data Luciano \& Næs (2009) considered PCA using the estimates obtained from a regular two-way ANOVA.

In this paper, we will be considering principal component analysis of d-prime values obtained from sensory discrimination tests using the binary paired comparison. We will consider two approaches; one analyzing the raw d-prime values, transforming data by the inverse of the psychometric function, and one where the d-prime values are obtained from analyzing data by a model. More specifically, Linander et al. (2019) introduced a Thurstonian mixed model for binary paired comparison data. This model can be used to analyze multiple sensory attributes one at a time. From each analysis, assessor-specific as well as product-specific d-prime values are obtained, and these will be used in a principal component analysis. Thus, information about products as well as assessors are obtained across sensory attributes when considering the principal component analysis.

In a world that constantly evolves, e.g. due to health initiatives, it is beneficial for companies to be able to compare a product with versions that are subtle modifications of this product by investigating whether any of the new variants have a similar sensory profile to the known product across many attributes.

Considering discrimination tests for subtle product differences, the 2-AFC test is much simpler than the 3-AFC, and almost as sensitive. Thus, 2-AFC is the preferred test to evaluate a specific sensory attribute (Dessirier \& O'Mahony, 1999; Van Hout et al., 2011; Ennis \& Jesionka, 2011). For PCA to be relevant, various sensory attributes must be evaluated for a group of products. Therefore, we extend the methodology for binary paired comparisons by describing how PCA can be used to evaluate d-prime values obtained from such studies. Moreover, the 2-AFC does not use a scale and will not lead to variability of using a scale. Furthermore, the 2-AFC enables a constant reference, thus providing a rather simple way to obtain relative sensory profiles. However, there are limitations associated with the use of 2-AFC that we will also address in this paper.

The methodology presented in this paper is a step towards building a bridge between discrimination tests and descriptive analysis by considering principal component analysis using d-prime values obtained from a discrimination test. PCA makes it possible to obtain knowledge about possible correlations among sensory attributes that would not have been detected if only univariate analyses were considered. Furthermore, the biplot presenting the results from the PCA gives an easy way to gain information about similarities between products across multiple sensory attributes. Additionally, it is possible to gain knowledge about the assessors, e.g. if obvious mistakes are made it would be possible to identify these in the assessor biplot.

The paper is structured such that we introduce PCA using d-prime values in Section 2. We consider PCA using model-based product d-prime values in Section 3 In Section 4, we consider PCA using assessor specific d-prime values. Section 5 presents important aspects to consider when doing PCA using product d-prime values. We conclude the paper with a discussion in Section 6

\section{PCA using d-prime values}

Principal component analysis is a well-known multivariate analysis used in many applications, including sensory science. Many books and papers exist, giving a good introduction to PCA e.g. Næs et al. (2010), Varmuza \& Filzmoser (2009), Bro \& Smilde (2014).

In this paper, principal component analysis is considered using d-prime values obtained from a sensory discrimination study. We consider discrimination studies using binary paired comparisons in the sense of unbounded 2-AFC tests where no correct answer exists. Furthermore, we assume that the comparisons are of the type product A vs. control, product B vs. control and so forth.

Throughout the paper, we use biplots to illustrate the results obtained from the PCA, since these show useful information in a nice way. Biplots can be used to illustrate relations among products and attributes as well as to obtain information about the individuals. Basically, a biplot shows the scores and loadings in a combined figure, under given conditions, making it possible to interpret features regarding products and attributes e.g. correlations among the attributes. The angle between two arrows can be used to get information about the correlations among the two attributes for the dimensions displayed in the biplot; an acute angle implies the attributes are positively correlated, an obtuse angle implies negatively correlated, and a right angle implies the attributes are uncorrelated. Furthermore, the length of the arrow approximates to the standard deviation of that attribute. Gabriel \& Odoroff (1990) gives a detailed description of interpreting biplots, and for a thorough description of the definition of a biplot, we refer the reader to 


\section{Gabriel (1971).}

Interpreting correlations is a fundamental part of understanding the phenomenon the sensory study is intended to investigate. Furthermore, understanding the correlations between attributes can be useful in the sense that information is gained about possible combinations of a product. e.g. if two attributes are positively correlated and one would like to increase one attribute while decreasing the other, a positive correlation indicates that this will be impossible.

One important aspect of the d-prime values is that they should be comparable such that two similar values ensure similar characteristics of the corresponding products. The d-prime values we consider in this paper are comparable, since the d-prime values can be both positive as well as negative, depending on the perception of the product relative to the control. Thus, positive values indicate that the product has a stronger intensity of the sensory attribute than the control. Similarly, negative values indicate a weaker intensity of the product.

\subsection{Scaling and centering}

In many applications, data are centered as well as scaled before doing the PCA. The scaling is usually important because variables can be measured using different scales. However, when considering variables within sensory panel studies, such differences in scales rarely occur. Thus, PCA is often applied without scaling the variables when considering experiments in sensory panel studies (Borgognone et al., 2001; Næs et al., 2010; Lawless \& Heymann, 1998). For the d-prime values, no scaling is used, since all of the values are on the same scale, namely the d-prime scale where the d-prime values will mostly lie in the interval from -3 to 3 when negative d-prime values are allowed as in the binary paired comparison. Therefore, with the same reasoning as in sensory science in general, no scaling is done using d-prime values in a principal component analysis.

In sensory science, as well as in many other applications, data are often centered when doing a PCA. This will also be the general approach in this paper. There might be situations where all (or a majority) of the d-prime values from a binary paired comparison are either positive or negative. In such situations, it is important to center the data when doing the principal component analysis. The impact of not centering is that all the sensory attributes will appear to be positively correlated, even when this is not reflecting the correct nature of the potential correlations among the sensory attributes. Thus, we recommend centering when using raw d-prime values for the PCA. With regard to the productspecific d-prime values from a Thurstonian mixed model, both centered and non-centered PCA are considered. Each of these makes it possible to get different types of information on the products. In Section 3.3 we will consider the specific situation where an interpretation exists using non-centered product-specific d-prime values.

\subsection{PCA using raw product d-prime values}

In this section, we consider PCA using raw product d-prime values that are found by transforming the data. Let $x_{i j}$ be the number of times product $i$ is chosen out of a total of $n_{i j}$ answers for the $j$ th sensory attribute. Let $p_{i j}=x_{i j} / n_{i j}$ be the proportion of times product $i$ is chosen for the $j$ th attribute. Data are transformed into d-prime values using the inverse of the psychometric function for the binary paired comparison:

$$
f_{\text {paired }}^{-1}\left(p_{i j}\right)=\Phi^{-1}\left(p_{i j}\right) \sqrt{2}=d_{i j}^{\prime}
$$

The psychometric function in (1) is used by many authors (e.g. Linander et al. (2019) and Brockhoff \& Christensen (2010)) but derived in earlier work by Ennis (1993) and dating all the way back to Thurstone's pioneering work (Thurstone, 1927). The d-prime values found by (1) are the so-called raw d-prime values that are used in the PCA. We illustrate this approach by an example.

We simulate binary paired comparison data to illustrate the advantages of doing a principal component analysis using raw d-prime values compared to considering univariate analyses. We impose structure among products and sensory attributes in the data in order to find this structure in the results of the PCA. The comparisons considered are of the type product vs. control and the simulated data are the number of times a product is chosen.

We consider 10 products and 6 sensory attributes for 25 assessors. We impose positive as well as negative correlations among the sensory attributes. More specifically, attributes 1, 2 and 3 are positively correlated and attribute 4 and attribute 5 are negatively correlated. Furthermore, attributes 4 and 6 are positively correlated.

We impose similarities and differences between the products. More specifically, products 1, 2 and 3 are similar and products 7,8 and 9 are similar but different to products 1,2 and 3. Products 4 and 5 are completely different as 
having low or high sensory intensity when the other product has the opposite sensory intensity. We refer the reader to Appendix A for more details regarding the simulation of the data. The d-prime values for the simulated data are shown in Table 1 . We use these values in a principal component analysis.

[Table 1 about here.]

We consider the centered PCA which results in the biplot shown in Figure 1

[Figure 1 about here.]

The arrows for attributes 4 and 6 are close together, implying that these two attributes are positively correlated. The same holds for attributes 1 and 3. Furthermore, the arrow for attribute 2 is pointing in the same direction as the arrows for attributes 1 and 3, implying that attribute 2 is also positively correlated with attributes 1 and 3. Additionally, the arrow for attribute 5 is pointing towards the opposite direction of the arrows for attributes 4 and 6 , implying that attribute 5 is negatively correlated with attributes 4 and 6 . These findings correspond to the structure we imposed on the data.

Products 1, 2 and 3 are close together, implying similarities between these products. Similarly, products 7 and 8 are also rather close, implying similarities between these two products. Furthermore, product 9 is not too far away from products 7 and 8 , and therefore it resembles these products. Additionally, products 4 and 5 are in opposite directions with respect to the first PC, implying differences among these two products. Furthermore, products 6 and 10 are the products with the strongest sensory intensity of attribute 5 . However, they are rather far from one another, as well as the other products, implying that they overall are different from the other products.

The findings regarding correlations among the sensory attributes would not have been detected if only univariate analyses were considered. Furthermore, the biplot gives an easy way to gain information about similarities between products across multiple sensory attributes.

\subsection{Discrimination study}

In this section, we describe an existing discrimination study that we use as an example in the remainder of this paper. This study is the same discrimination study as is used in Linander et al. (2019). We briefly explain the structure of the discrimination study and refer the reader to Linander et al. (2019) for further details.

The overall aim of this study was to find a new product that has some of the same sensory characteristics as an existing product. In this study, the assessors were comparing different test products with the same control product. A sample of a test product as well as a sample of the control product were applied to an assessor's own skin. The assessor had to choose the sample with the strongest intensity of the attribute in question.

The study included eight test products assessed by a maximum of 25 assessors. Not all assessors evaluated all the test products. The assessors evaluated eight attributes, five of these were assessed immediately after application to the skin. In addition, three of these attributes were re-assessed 5 minutes after application. Each assessor evaluated each test product twice by making one comparison in two consecutive sessions. Thus, the maximum number of assessments for each test product is 50 . The number of evaluations for the test products range from 40 to 46 .

The desired sensory characteristics for the new product is to be less sticky and at the same time not to be greasier than the control product. Furthermore, the new product should be at least as silky as the control product, though preferably silkier. This will be evaluated by observing the sensory profile for the products in the biplots.

\section{PCA using product-specific d-prime values obtained from a Thurstonian mixed model}

In Section 2.2, the PCA was applied using a set of d-prime values found by using the inverse of the psychometric function. In this section, we will model the probabilities of a product being chosen. The d-prime values obtained from such a model will then be analyzed by PCA. It is not necessary to fully understand the model to be able to comprehend the results of the PCA of the d-prime values. Thus, for readers without interest in how the model is defined, Section 3.1 can be omitted. 


\subsection{Thurstonian Mixed Model}

In this section, we describe the Thurstonian mixed model (Linander et al. 2019) that we use to find the d-prime values for the PCA. The Thurstonian mixed model analyzes data for one sensory attribute at a time. Thus, to obtain the results for all sensory attributes in the data, the analysis must be conducted multiple times.

The Thurstonian mixed model models data obtained from binary paired comparison studies with comparisons like product A vs. control, product B vs. control and so forth. Each observation is binomially distributed:

$$
Y_{l m k} \sim \operatorname{binomial}\left(p_{l m}, 1\right)
$$

where $l=1, \ldots, L$ represents the products, $m=1, \ldots, n_{l}$ represents the assessors for the $l$ th product and $k=$ $1, \ldots, K$ represents the sessions ( $K=2$ and $L=8$ for the discrimination study described in Section 2.3). We assume that $p_{l m}$, the probability of the $m$ th assessor choosing the $l$ th product, is independent of the sessions:

$$
p_{l m}=P\left(Y_{l m k}=1\right)
$$

It is possible to impose a linear structure of $p_{l m}$ which explains the variables that are affecting these probabilities. We consider a model where the probabilities are explained by products as well as assessors:

$$
p_{l m}=f_{\text {paired }}\left(\mu+\alpha_{l}+b_{m}\right)
$$

where $f_{\text {paired }}$ is the psychometric function with its inverse given in (1). Additionally, $\mu$ is the overall average difference between the products and the control and $\alpha_{l}$ is the difference for the $l$ th product to the average product-difference $\mu$. Thus, the sensory difference for the $l$ th product to the control is

$$
\delta_{l}=\mu+\alpha_{l}
$$

Furthermore, $b_{m}$ is the random effect of the $m$ th assessor where $b_{m} \sim N\left(0, \sigma_{m}^{2}\right)$ which are independent for all $m$. $b_{m}$ is the difference for the $m$ th assessor to the average product-difference $\mu$ on the d-prime scale. Thus, the sensory difference, on the d-prime scale, between the products and the control for the $m$ th assessor is $\tilde{b}_{m}=\mu+b_{m}$. We refer the reader to Linander et al. (2019) for further details regarding the Thurstonian mixed model.

The results from the Thurstonian mixed model that we use in this paper are product-specific as well as assessor-specific d-prime values for all of the sensory attributes.

\subsection{Centering}

\subsubsection{Background}

An important aspect of PCA is whether or not to center the data before doing PCA. For the product-specific dprime values, both situations will be considered, since each of these contributes valuable information regarding the products. As we will show below, when centering the product-specific d-prime values, the information regarding the control is removed. However, when the d-prime values are used without centering, the information about the control is maintained in the PCA.

To be able to distinguish the estimates of the product-specific d-prime values obtained for the different sensory attributes, an additional sub-script will be used:

$$
d_{l j}^{\prime}=\hat{\mu}_{j}+\hat{\alpha}_{l j}, \quad l=1, \ldots, L
$$

where $j=1, \ldots, J$ represents the sensory attribute and $\hat{\mu}_{j}$ and $\hat{\alpha}_{l j}$ are the estimates obtained from the analysis of the $j$ th attribute. Thus, $d_{l j}^{\prime}$ is the sensory difference for the $l$ th product to the control for the $j$ th sensory attribute.

The model in (2) is over-parameterized, thus it is assumed that for each $j$ :

$$
\sum_{l=1}^{L} \hat{\alpha}_{l j}=0
$$


When centering the product-specific d-prime values, the mean value of the $d_{i j}^{\prime}$ s for each $j$ is subtracted. $\bar{d}_{. j}^{\prime}$; the mean value over $i$, for a given $j$, reads:

$$
\begin{aligned}
\bar{d}_{. j}^{\prime} & =\frac{1}{L} \sum_{l=1}^{L} d_{l j}^{\prime} \\
& =\frac{1}{L} \sum_{l=1}^{L}\left(\hat{\mu}_{j}+\hat{\alpha}_{l j}\right) \\
& =\frac{1}{L} L \hat{\mu}_{j}+\frac{1}{L} \sum_{l=1}^{L} \hat{\alpha}_{l j} \\
& =\hat{\mu}_{j}
\end{aligned}
$$

where the last equality follows from (3). Therefore, the centered d-prime values are given as:

$$
\begin{aligned}
d_{i j}^{\prime}-\bar{d}_{. j}^{\prime} & =d_{i j}^{\prime}-\hat{\mu}_{j} \\
& =\left(\hat{\mu}_{j}+\hat{\alpha}_{l j}\right)-\hat{\mu}_{j} \\
& =\hat{\alpha}_{l j}
\end{aligned}
$$

Thus, when doing the PCA using the centered d-prime values, the $\hat{\alpha}_{l j} \mathrm{~s}$ are used. Hence, when interpreting the results of the PCA, the information regarding the control has been removed. Recall that $\alpha_{l j}$ merely expresses the difference from the $l$ th product to the average product-difference $\mu_{j}$ for the $j$ th attribute. Thus, when considering the PCA on the centered d-prime values, it is possible to compare the products with each other, but not with the control.

\subsubsection{Example}

In this section, we consider the centered PCA using the data from the discrimination study described in Section 2.3. More specifically, we consider the product-specific d-prime values which are obtained from model (2) and listed in Table 2 . Note that the d-prime value for product $H$ for Silky (0 minutes) equals $-\infty$. We have chosen to reanalyze data with a 0 changed to 1 for one assessor to obtain a finite value. We believe this is a sensible choice for handling the extreme value, since the lowest number of evaluations for Silky is 40 . Thus, the imputed value expresses a rather large difference between $\mathrm{H}$ and the control. This approach results in an imputed value of -3.32 for product $\mathrm{H}$ for Silky (0 minutes). Several approaches exist for handling extreme values, and we will address some of these in Section 5.1

[Table 2 about here.]

The PCA using the centered product-specific d-prime values results in the biplot shown in Figure 2

[Figure 2 about here.]

The first principal component, PC1, is explained by Sticky and Silky initially, as well as after five minutes, for both attributes. Test products $\mathrm{H}$ and $\mathrm{D}$ are placed in opposite directions with respect to $\mathrm{PC} 1$; $\mathrm{H}$ is stickier and less silky than D. Test products A and G are positioned close to each other with respect to PC1. Thus, A and G have similar sensory properties regarding stickiness and silkiness. The second principal component, $\mathrm{PC} 2$, is primarily explained by Thickness. Test products $\mathrm{A}$ and $\mathrm{G}$ are the thickest products whereas $\mathrm{H}$ is the least thick product. Furthermore, test products B, F, E, C and D are similar with respect to Thickness. In conclusion, test products $A$ and $G$ are similar with respect to the sensory attributes Thickness, Silky and Sticky initially and after 5 minutes for the two latter attributes. Furthermore, test product $\mathrm{H}$ is very different from all of the other test products. Additionally, test product $\mathrm{D}$ is by far the most silky test product. 


\subsection{No centering \\ 3.3.1. Background}

When the d-prime values are not centered, the information about the control is maintained. The d-prime values that are used are the $\hat{\mu}_{j}+\hat{\alpha}_{l j}$ s. The difference $\hat{\mu}_{j}+\hat{\alpha}_{l j}$ is the estimated sensory difference between the $l$ th product and the control for the $j$ th sensory attribute. Therefore, all the d-prime values used for the PCA are differences between the products and the control. Thus, the origin in the biplot corresponds to the control. Hence, when differences between products and the control are of interest, the non-centered PCA will provide insight about these.

Before doing a non-centered PCA, it is important to consider whether the set of d-prime values is suitable for this type of analysis. As written in Section 2.1, a non-centered PCA only works if the d-prime values are positive as well as negative. Otherwise, this analysis risks introducing correlations that are not necessarily there. It can be difficult to determine whether the d-prime values are scattered such that enough positive and negative values occur. For now, we do not have any specific recommendations, only that one must look at the signs of the d-prime values to decide whether it seems reasonable to do the non-centered PCA. Thus, future research could investigate whether it is possible to determine more specific guidelines regarding the distribution of the d-prime values.

\subsubsection{Example}

In this section, we consider the non-centered PCA using the data from the discrimination study described in Section 2.3. As in Section 3.2.2 we use the imputed value of -3.32 instead of the extreme value. Considering the d-prime values in Table 2, a number of positive as well as negative values are occurring. Thus, we believe that it is sensible to consider the non-centered PCA. The biplot for the PCA using the non-centered product-specific d-prime values is shown in Figure 3

\section{[Figure 3 about here.]}

The arrows for Thickness and Absorption are short. That, in combination with many small d-prime values, makes it extremely difficult to conclude anything regarding Thickness and Absorption from Figure 3/(the sign of the values will easily change, since other attributes will affect the values of the scores more heavily). Considering Greasy (initially and after 5 minutes), all of the test products are less greasy than the control, since they are placed opposite the direction of the arrows for Greasy.

Generally, when interpreting the biplot from a non-centered PCA, we use the perpendicular line for each attribute; the straight line going through the origin that is orthogonal to the arrow for the attribute in question. For simplicity, we have only included the perpendicular line for one attribute ( $\mathrm{Si} \perp \mathrm{ky}$ ) as an illustration. However, it is possible to obtain the perpendicular lines for all attributes using the dot product. The perpendicular line is used to determine the relation between the product and the control for that attribute. Thus, for a product to have similar intensity as the control, for a given sensory attribute, the product should be positioned closely to the imaginary/perpendicular line. For a product to have a stronger sensory intensity of the attribute in question, it should be closer to the tip of the arrow than the control. Similarly, for a product to have a weaker sensory intensity than the control, it should be further away from the tip of the arrow than the control.

The distance to the perpendicular line for the product to have similar intensity depends on the specific objective of the study. Thus, it is up to each sensory scientist to evaluate whether a product is close enough to the perpendicular line for that product and the control to have similar sensory intensities.

With respect to silkiness, the majority of the test products are less silky than the control. Furthermore, it appears that test product $\mathrm{C}$ is just about as silky (initially) as the control, and test product $\mathrm{D}$ is the only test product which clearly is silkier than the control. Considering stickiness, test products $\mathrm{G}$ and $\mathrm{H}$ are stickier (initially) than the control, and $\mathrm{A}$ is more or less as sticky (initially) as the control. It appears that the remaining test products are less sticky (initially) than the control. Furthermore, it appears that the only test product which is stickier than the control after 5 minutes is test product $\mathrm{H}$.

It is possible, from Figure 3, to identify the most interesting test products based on the most important sensory properties. These properties are pre-specified by the sensory scientist and they are not chosen based on the results from the PCA. Recall that a test product is interesting when it is as silky as the control as well as being less greasy and sticky. Thus, the most interesting test products are $C$ and $D$ with $D$ being the more promising of the two since it is silkier than $\mathrm{C}$. It should be noted that the desired characteristics are decided by the sensory scientist (e.g. when designing the 
study). Thus, for other studies other sensory attributes might be the most important.

\section{PCA using assessor-specific d-prime values from a Thurstonian mixed model}

Individual differences are important and relevant to investigate in relation to sensory studies, and for QDA data many methods exist enabling this information. In this section, we show how information about assessors can be obtained considering PCA using assessor-specific d-prime values from a multi-attribute 2-AFC study.

To gain knowledge about which assessors are proportionally choosing products similarly on average across products, the $b_{m} \mathrm{~s}$ are considered for each attribute. To be able to distinguish the estimates obtained for the different attributes, an additional sub-script will be used. Thus, $b_{m j}$ is the difference for the $m$ th assessor to the average product-difference $\mu_{j}$, on the d-prime scale, for the $j$ th attribute. The centered and non-centered PCA will give similar results, since $\mathrm{E}\left(b_{m j}\right) \approx 0$, thus the assessor-specific d-prime values $b_{m j} \mathrm{~s}$ are almost centered. Considering the $b_{m j} \mathrm{~s}$, it is possible to investigate how the assessors proportionally choose products compared to the average.

\subsection{Example}

In this section, we consider PCA using the data from the discrimination study described in Section 2.3 More specifically, we consider the assessor-specific d-prime values obtained from model (2). When testing skin care products, it is important to capture individual subject differences, since people have different skin types which respond differently to such products.

\section{[Figure 4 about here.]}

The biplot for the assessor-specific d-prime values is shown in Figure 4 The first principal component is explained by Silky, mostly evaluated after five minutes, but also initially. The assessors 7 and 8 are proportionally choosing products the most with respect to silkiness. Assessor 19 is the assessor proportionally choosing the control the most regarding Silky. The second principal component is mostly explained by Greasy (initially and after five minutes). Assessor 23 is the assessor proportionally choosing products the most with respect to Greasy. There are assessors who are proportionally choosing products similarly on average across products. The assessors 12 and 15 are close in the biplot. Assessors 22, 18 and 11 are also close together. Furthermore, it appears that the assessors in the lower left quadrant have a tendency to proportionally choose products more than the average assessor across the attributes, since all of the arrows points toward left and/or down.

In our opinion, Figure 4 can be used to look for patterns of the assessors' way of selecting products which might be missed otherwise. However, this is not the same as being able to interpret the quality of an assessor. We believe that to be able to interpret the quality of the assessors, prior knowledge about the 'correct' product differences must be available.

When the arrow is short, it was easier for the assessors to evaluate that attribute compared to the other attributes. However, it is not possible, from the biplot, to explain whether this is a consequence of the attribute being easier to assess or whether the products were more differentiable with respect to this attribute.

The correlations among the attributes can be used to look for obvious misunderstandings among the assessors in the evaluation of the attributes, e.g. if two attributes that are positively correlated are shown to be negatively correlated, this could indicate that more training of the panel is needed. Furthermore, if some correlations appear to be counterintuitive, this could be a result of the assessors misunderstanding how to evaluate some of the attributes or simply because they find evaluating these attributes difficult.

The center of the biplot corresponds to an average assessor. The further away from the center an assessor is positioned, the more different assessments from that assessor are compared to an average assessor. If all the assessors are close to the center, the assessors are assessing similarly. The more the assessors are spread out, the more diverse the assessors are assessing across products. Thus, when interpreting the biplot of the assessor-specific d-prime values, information is obtained about how variable the assessors were in the study. Therefore, insights are achieved into how the product averages are obtained. Often, the aim of a panel is to include a diverse group of assessors, and in such situations, it is to be expected that the assessors will be variable. However, if the panel consists of similar assessors, e.g. assessors with the same skin type, then it is likely that the assessors will be less variable. Thus, whether the variability of 
assessors could be an issue depends on the given objective of a study.

The interpretation of Figure 4 gives the idea of interpreting such a biplot. However, in this specific discrimination study we have a limitation of not all assessors evaluating all products. Thus, some of the differences seen in Figure 4 might be due to this design issue. Thus, when considering the biplot for the assessors, preferably all assessors should evaluate all products.

\section{Considerations regarding PCA using product d-prime values}

In this section, we describe important aspects to consider when analyzing product d-prime values with PCA.

\subsection{Handling extreme values}

As was occurring in Section 3.2.2 there is a likelihood of observing d-prime values equal to $\pm \infty$. In this section, we suggest approaches for handling such extreme values. We consider PCA using the so-called raw d-prime values as well as model-based d-prime values. Some approaches handling the extreme values are applicable for both types of d-prime values. Other solutions only work for one or the other type. An approach which only works for the raw d-prime values, is to use the proportion $1 / n$ rather than $0 / n$ (or $(n-1) / n$ rather than $n / n$ ) when finding the d-prime values. Changing the proportion ensures finite d-prime values. However, this raises the question of how large $n$ should be before this change of proportion is meaningful. For small $n$, this change will imply a completely different result, e.g. if $n=2$ then $x=0$ results in a d-prime value of minus infinity, whereas $x=1$ results in a d-prime value of 0 . For $n=50$, the proportion $1 / n$ corresponds to a d-prime value of -2.9 , which is still expressing a large difference between the products. For this approach to be reasonable, a rather large $n$ is needed and a topic for future research is to investigate when $n$ is 'large enough'. An equivalent approach for the model-based d-prime values is to change one data point from 0 to 1 and then re-analyze data by the Thurstonian mixed model to find the value to replace the extreme value. Using this approach, it is important to only extract the imputed value from the new analysis.

Another approach is to use a different proportion than $1 / n$ when imputing a value for the raw d-prime values. It might be possible to weight the data in a better way compared to using $1 / n$ considering the raw d-prime values. Thus, an issue for future research is to investigate whether it is possible to claim that, in general, one weight is better than another.

Other approaches valid for both types of d-prime values, are to either remove the sensory attribute(s) with extreme values or to remove the product(s) with extreme values. Obviously, these approaches only work if not too many different attributes or products have extreme values. However, if there are many extreme values, one could argue whether the PCA is meaningful to consider at all.

Yet another approach is to choose an arbitrary value that expresses a large difference between the products, and use that instead of the extreme value. Our preliminary findings, considering the data from the discrimination study described in Section 2.3. indicate that with only one extreme value the results of the PCA seem to be affected as expected when changing the size of the imputed value; the more extreme the imputed value, the more extreme the allocation of that product for the attribute the value is imputed for becomes, without affecting the interpretation regarding the remaining products as well as attributes.

It is important to carefully consider how to handle the extreme values and future research could investigate in more detail how different approaches affect the results of the PCA.

\subsection{Product Selection}

As described in Section 2.1 it is important that the d-prime values used for PCA are positive as well as negative. When designing a discrimination study, it is possible to minimize the risk of getting clustered d-prime values in the way the products are selected. In the case of an extreme control, the likelihood of getting d-prime values with the same sign increases compared to having a non-extreme control.

Having d-prime values with the same sign particularly becomes an issue when considering the non-centered PCA in Section 3.3. This approach is only valid when a few of the attributes have d-prime values with the same sign.

Thus, considering binary paired comparisons to be used in PCA, preferably a non-extreme control should be used. Currently, we do not have a definition of when a control is extreme vs. non-extreme. However, it would be relevant 
to investigate whether it is possible to determine when a control is non-extreme. Furthermore, it would be interesting to investigate how the results from the centered PCA are affected by an increasing number of attributes with values of the same sign. Additionally, it would be interesting to investigate whether it is possible to find a cut-off value for the percentage of attributes with an extreme control where the results are affected such that they no longer reflect the correct nature of data.

\section{Summary and discussion}

In this paper we have presented a first step towards building a bridge between descriptive analysis and discrimination testing. More specifically, we have suggested a way to gain knowledge about products and assessors across sensory attributes by considering d-prime values obtained from a binary paired comparison, and we have used these in a principal component analysis. As written in Section 1 insights about products and individual differences are obtained when considering a multi-attribute 2-AFC study. Furthermore, the binary paired comparison is preferable when considering subtle product differences due to its simplicity as well as the lack of a scale in this discrimination test. Additionally, the 2-AFC test provides a constant reference giving relative sensory profiles. However, to the best of our knowledge, it has not been documented in the literature whether increased sensitivity will be obtained in a multi-attribute 2-AFC study. It is beyond the scope of this paper to investigate this, but it would be interesting to compare the sensitivity for QDA with the sensitivity for a multi-attribute 2-AFC study as a part of future research on this topic.

When considering PCA using d-prime values, various aspects are important to consider carefully in order to ensure a valid interpretation of the results. We have addressed the importance of centering the d-prime values to ensure that we do not force incorrect correlations between the sensory attributes. There is one exception, where it is valid to consider the non-centered PCA; using the product-specific d-prime values from a Thurstonian mixed model given that both positive and negative d-prime values are present in the data.

An interesting continuation is to investigate whether it is possible to determine guidelines for a distribution of positive and negative d-prime values.

Another important aspect to consider is how to handle extreme values. We have suggested different ways of dealing with extreme values, although more work is needed to figure out in more detail how different approaches affect the results of the PCA.

Additionally, we have dealt with how to choose the product used as the control in the evaluations; it is important to choose a non-extreme control. Currently, we do not have a definition of when a control is non-extreme, and it would be relevant to investigate the possibility of making such a definition. An interesting continuation of the work presented in this paper is to consider other types of paired comparisons such as product $\mathrm{A}$ vs product $\mathrm{B}$, product $\mathrm{B}$ vs product $\mathrm{C}$ and so forth. Furthermore, it would be interesting to consider other ways of obtaining the d-prime values than considering binary paired comparisons. As mentioned in Section 2, it is important that the d-prime values are comparable. Thus, it is important to ensure that this is fulfilled when investigating whether it is possible to use d-prime values that are not obtained from a binary paired comparison. However, further research is needed to comprehend the impact of how the d-prime values are obtained.

In order to facilitate easy choices regarding similarities between products and control, it would be relevant to investigate whether a d-prime interpretation of distances exists in the biplot.

Future research regarding the biplot for the assessor d-prime values could be to investigate patterns if additional information is available for the sensory scientist, e.g. whether the assessors are clustered according to their skin type. Furthermore, it would be relevant to consider whether it is generally possible to identify good and bad assessors.

As this paper is aimed at being a first step in building a bridge between descriptive analysis and discrimination testing, it would be relevant to continue building this bridge in the future. One way to continue this work is by comparing the performance of principal component analysis of data from descriptive analysis studies with the performance of PCA using d-prime values from the binary paired comparison.

\section{Acknowledgments}

The research that led to this paper is funded by the Technical University of Denmark and Unilever U.K. Central Resources Limited. Unilever also provided the data used as an example in sections 3 and 4 . Furthermore, the authors 
would like to thank the editor as well as two anonymous reviewers for comments that led to a substantial improvement of this paper. Additionally, the first author would like to thank Thomas Kallemose for many rewarding discussions.

\section{Appendix A. Details regarding the example in Section 2.2}

In this appendix, we describe in more detail how we simulated the data used in Section 2.2 The comparisons considered are all of the type product vs. control in a binary paired comparison. Thus, the simulated data values are the number of times a product is chosen for each comparison of a product with the control.

The number of times product $i$ is chosen for attribute $j$ is binomially distributed:

$$
Y_{i j} \sim \operatorname{binomial}\left(n_{i j}, p_{i j}\right)
$$

where $n_{i j}$ is the total number of evaluations and $p_{i j}$ is the probability that the $i$ th product is chosen for the $j$ th sensory attribute. Since we are simulating binomial data, we impose the structure among products and attributes on the probabilities for a product being chosen. We consider 10 products and 6 sensory attributes for 25 assessors. Thus, $i=10$ and $j=6$.

The attributes 1,2 and 3 are made positively correlated by having the same probabilities for all products:

$$
p_{i 1}=p_{i 2}=p_{i 3} \quad i=1, \ldots, 10
$$

Attribute 4 and attribute 5 are negatively correlated, one having high probabilities where the other has low probabilities:

$$
p_{i 5}=1-p_{i 4} \quad i=1, \ldots, 10
$$

Additionally, attributes 4 and 6 are positively correlated:

$$
p_{i 4}=p_{i 6} \quad i=1, \ldots, 10
$$

Products 1, 2 and 3 are made similar as having the same probabilities:

$$
p_{1 j}=p_{2 j}=p_{3 j} \quad j=1, \ldots, 6
$$

Similarly, products 7,8 and 9 are similar but with a different probability than products 1,2 and 3 :

$$
p_{7 j}=p_{8 j}=p_{9 j} \neq p_{1 j} \quad j=1, \ldots, 6
$$

Products 4 and 5 are made opposite in the sense where one has high probabilities the other has low probabilities:

$$
p_{5 j}=1-p_{4 j} \quad j=1, \ldots, 6
$$

The probabilities that we used to simulate the data for the example in Section 2.2 are shown in Table 3

[Table 3 about here.]

The simulated data, shown in Table 4 , are transformed into d-prime values using:

$$
f_{\text {paired }}^{-1}\left(p_{i j}\right)=\Phi^{-1}\left(p_{i j}\right) \sqrt{2}=d_{i j}^{\prime}
$$

where the $p_{i j} \mathrm{~s}$ are the proportions of the data corresponding to each entry of Table 4 being divided by 25 .

[Table 4 about here.] 


\section{References}

Borgognone, M. G., Bussi, J., \& Hough, G. (2001). Principal component analysis in sensory analysis: covariance or correlation matrix? Food Quality and Preference, 12, 323-326.

Bro, R., \& Smilde, A. K. (2014). Principal component analysis. Analytical Methods, 6, 2812-2831.

Brockhoff, P. B., \& Christensen, R. H. B. (2010). Thurstonian models for sensory discrimination tests as generalized linear models. Food Quality and Preference, 21, 330-338.

Dessirier, J.-M., \& O'Mahony, M. (1999). Comparison of d' values for the 2-afc (paired comparison) and 3-afc discrimination methods: Thurstonian models, sequential sensitivity analysis and power. Food Quality and Preference, 10, $51-58$.

Ennis, D. M. (1993). The power of sensory discrimination methods. Journal of Sensory Studies, 8, 353-370.

Ennis, J. M., \& Jesionka, V. (2011). The power of sensory discrimination methods revisited. Journal of Sensory Studies, 26, 371-382.

Gabriel, K. (1971). The biplot graphic display of matrices with application to principal component analysis. Biometrika, 58, 453-467.

Gabriel, K., \& Odoroff, C. L. (1990). Biplots in biomedical research. Statistics in medicine, 9, 469-485.

Lawless, H. T., \& Heymann, H. (1998). Sensory Evaluation of Food. Springer.

Linander, C. B., Christensen, R. H. B., Cleaver, G., \& Brockhoff, P. B. (2019). Individual differences in replicated multi-product experiments with thurstonian mixed models for binary paired comparison data. Food Quality and Preference, 75, 220-229.

Luciano, G., \& Næs, T. (2009). Interpreting sensory data by combining principal component analysis and analysis of variance. Food Quality and Preference, 20, $167-175$.

Næs, T., Brockhoff, P. B., \& Tomic, O. (2010). Statistics for Sensory and Consumer Science. Wiley.

Næs, T., \& Risvik, E. (1996). Multivariate analysis of data in sensory science. Elsevier.

Thurstone, L. (1927). A law of comparative judgment. Psychological Review, 34, 273-286.

Van Hout, D., Hautus, M. J., \& Lee, H.-S. (2011). Investigation of test performance over repeated sessions using signal detection theory: comparison of three nonattribute-specified difference tests 2-afcr, a-not a and 2-afc. Journal of Sensory Studies, 26, 311 - 321.

Varmuza, K., \& Filzmoser, P. (2009). Multivariate Statistical Analysis in Chemometrics. CRC Press. Taylor \& Francis Group. 


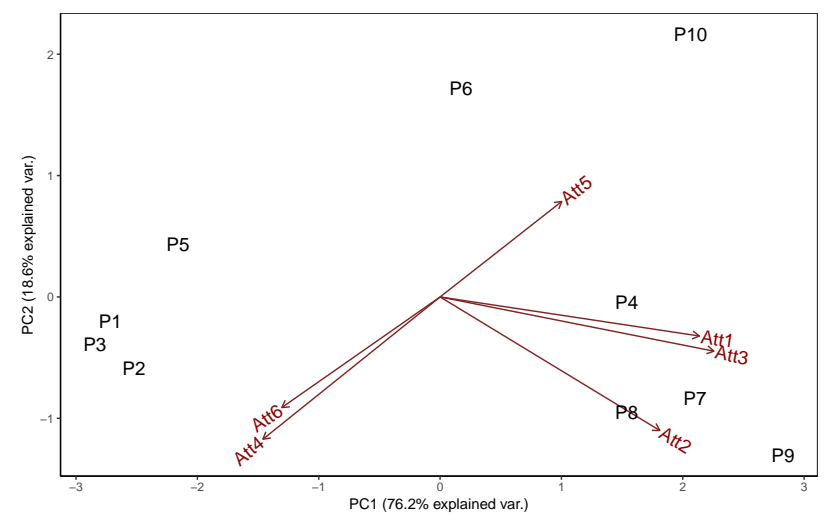

Figure 1: The biplot for the centered d-prime values from the simulated data. 


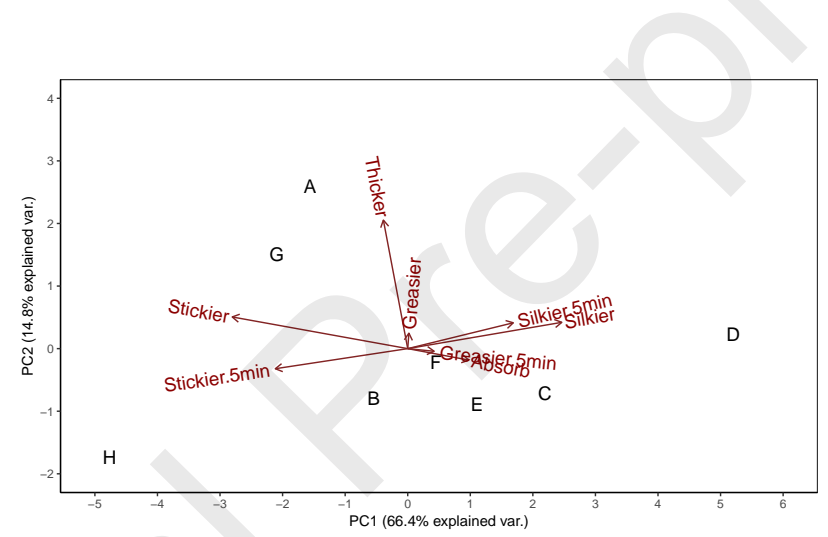

Figure 2: The biplot for the centered product specific d-prime values. 


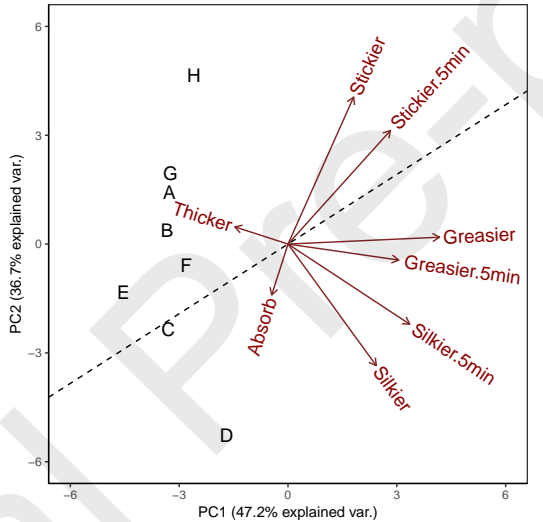

Figure 3: The biplot for the non-centered product-specific d-prime values. The dashed line is perpendicular to the arrow associated with Silkier. 


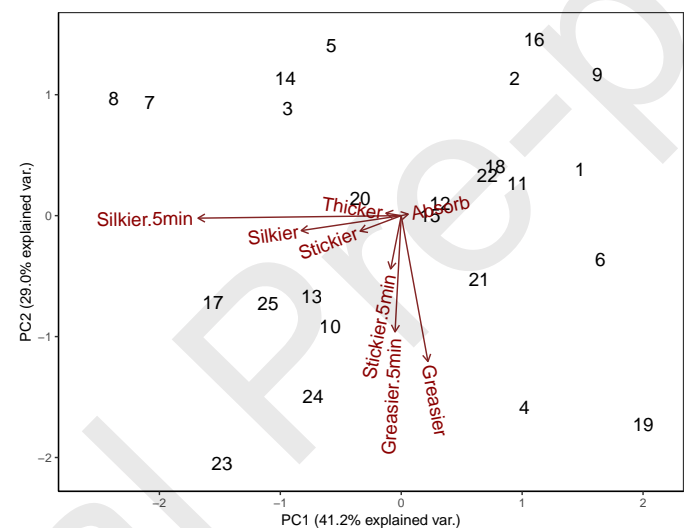

Figure 4: The biplot for the centered assessor specific d-prime values $b_{j}$. 
Table 1: Simulated d-prime values for the binary paired comparison.

\begin{tabular}{|c|cccccc|}
\hline Product & \multicolumn{7}{|c|}{ Attribute } \\
& 1 & 2 & 3 & 4 & 5 & 6 \\
\hline 1 & -1.00 & -0.82 & -0.82 & 1.41 & -0.07 & 0.66 \\
2 & -1.00 & -0.51 & -0.51 & 0.66 & -1.19 & 1.00 \\
3 & -1.41 & -0.36 & -1.00 & 1.19 & -0.51 & 0.66 \\
4 & 0.82 & 1.19 & 1.41 & -0.82 & 0.51 & -0.51 \\
5 & -0.51 & -0.51 & -1.41 & 0.21 & -0.36 & 0.21 \\
6 & -0.07 & -0.21 & 0.07 & -1.19 & 0.51 & -1.41 \\
7 & 1.66 & 1.41 & 1.99 & -0.36 & 0.07 & -0.51 \\
8 & 1.00 & 1.99 & 1.19 & -0.21 & 0.07 & -0.51 \\
9 & 1.99 & 2.48 & 1.99 & -0.21 & 0.51 & -0.66 \\
10 & 1.00 & 0.07 & 1.19 & -1.99 & 1.66 & -1.66 \\
\hline
\end{tabular}


Table 2: Product specific d-prime values found by using the model given in (2).

\begin{tabular}{|c|cc|cc|cc|c|c|}
\hline Test Product & \multicolumn{2}{|c|}{ Sticky } & \multicolumn{2}{|c|}{ Greasy } & \multicolumn{2}{c|}{ Silky } & Thickness & Absorption \\
& $0 \mathrm{~min}$ & $5 \mathrm{~min}$ & $0 \mathrm{~min}$ & $5 \mathrm{~min}$ & $0 \mathrm{~min}$ & $5 \mathrm{~min}$ & $0 \mathrm{~min}$ & $0 \mathrm{~min}$ \\
\hline A & 0.64 & -1.36 & -1.19 & -1.76 & -1.15 & -1.57 & 2.94 & -1.35 \\
B & -1.44 & 0.08 & -1.98 & -0.51 & -1.47 & -2.39 & 0.58 & 1.50 \\
C & -2.60 & -2.15 & -1.79 & -1.22 & -0.03 & -0.89 & -0.13 & 0.46 \\
D & -3.42 & -3.47 & -0.67 & -0.65 & 2.28 & 0.83 & -0.01 & 0.88 \\
E & -1.93 & -2.22 & -2.74 & -1.80 & -1.02 & -1.47 & -0.04 & 0.76 \\
F & -0.40 & -1.88 & -2.21 & -1.19 & -0.64 & -0.37 & -0.09 & -0.47 \\
G & 0.70 & -0.01 & -1.81 & -1.21 & -1.89 & -1.77 & 2.43 & 0.99 \\
H & 2.06 & 0.88 & -1.04 & -1.82 & $-I n f$ & -3.03 & -1.09 & -1.58 \\
\hline
\end{tabular}


Table 3: Probabilities used when simulating the binary paired comparison data.

\begin{tabular}{|c|cccccc|}
\hline Product & \multicolumn{7}{|c|}{ Attribute } \\
& 1 & 2 & 3 & 4 & 5 & 6 \\
\hline 1 & 0.30 & 0.30 & 0.30 & 0.70 & 0.30 & 0.70 \\
2 & 0.30 & 0.30 & 0.30 & 0.70 & 0.30 & 0.70 \\
3 & 0.30 & 0.30 & 0.30 & 0.70 & 0.30 & 0.70 \\
4 & 0.80 & 0.80 & 0.80 & 0.30 & 0.70 & 0.30 \\
5 & 0.20 & 0.20 & 0.20 & 0.70 & 0.30 & 0.70 \\
6 & 0.50 & 0.50 & 0.50 & 0.20 & 0.80 & 0.20 \\
7 & 0.90 & 0.90 & 0.90 & 0.40 & 0.60 & 0.40 \\
8 & 0.90 & 0.90 & 0.90 & 0.40 & 0.60 & 0.40 \\
9 & 0.90 & 0.90 & 0.90 & 0.40 & 0.60 & 0.40 \\
10 & 0.70 & 0.70 & 0.70 & 0.10 & 0.90 & 0.10 \\
\hline
\end{tabular}


Table 4: The simulated number of times a product was chosen.

\begin{tabular}{|c|cccccc|}
\hline Product & \multicolumn{7}{|c|}{ Attribute } \\
& 1 & 2 & 3 & 4 & 5 & 6 \\
\hline 1 & 6 & 7 & 7 & 21 & 12 & 17 \\
2 & 6 & 9 & 9 & 17 & 5 & 19 \\
3 & 4 & 10 & 6 & 20 & 9 & 17 \\
4 & 18 & 20 & 21 & 7 & 16 & 9 \\
5 & 9 & 9 & 4 & 14 & 10 & 14 \\
6 & 12 & 11 & 13 & 5 & 16 & 4 \\
7 & 22 & 21 & 23 & 10 & 13 & 9 \\
8 & 19 & 23 & 20 & 11 & 13 & 9 \\
9 & 23 & 24 & 23 & 11 & 16 & 8 \\
10 & 19 & 13 & 20 & 2 & 22 & 3 \\
\hline
\end{tabular}

